\title{
NUMERICAL INVESTIGATION OF VERTICAL GAS-LIQUID SEPARATORS USING COMPUTATIONAL FLUID DYNAMICS AND STATISTICAL TECHNIQUES
}

\author{
JULIO CESAR MENDEZ ${ }^{1}$, AHMET ERGIN EFENDIOGLU² \& JOSE LEONARDO GUEVARA ${ }^{3}$ \\ ${ }^{1}$ Mechanical Engineering Department, North Carolina A\&T State University, USA \\ ${ }^{2}$ Project \& Manufacturing Department, Turkish Petroleum Corporation (TPAO), Turkey \\ ${ }^{3}$ School of Mining and Petroleum Engineering, University of Alberta, Canada
}

\begin{abstract}
Many industrial devices found in the oil and gas industries are designed using empirical correlations, such as gas-liquid vertical separators. However, the physics involved in these devices are quite complex including multiphase flows and internal devices that are not considered in the empirical approach. Therefore, important discrepancies are found in industrial fields. This research conducts a numerical study using Computational Fluid Dynamics (CFD) to assess the different empirical models used in such designs. A short review of the different models is presented and compared and computational experiments are used to evaluate the parameters of importance of these devices. In addition, statistical models are implemented to evaluate the influence of operational parameters, properties of fluids and geometric variables to the efficiency of the separator and pressure drop. To this end, a surrogate model is developed using Kriging interpolation that allows evaluation of the different combination of parameters without running each design using CFD. Preliminary results demonstrate that the standard accepted as general reference ANSI/ANSI/API-12J provides the lowest efficiency and the higher pressure drop, albeit small, compared to the other methods.
\end{abstract}

Keywords: vertical oil-gas separator, CFD, optimization, efficiency of separation.

\section{INTRODUCTION}

Multiphase separator units are critical components in the oil-gas industry. Typically, they are followed by more critical devices such as heat exchangers, compressors, pumps and distillations columns. Therefore, understanding the separation process and providing an efficient design is important to save operational cost and increase reliability in processes. However, there is no analytical solution to describe such phenomenon and the available procedures are based on complex empirical correlations. Because of this, Computational Fluid Dynamics (CFD) has become an appropriate tool to gain insight into this phenomenon; an extensive literature review is shown in [1]. In a more recent research article, Efendioglu et al. [2] validated the international standard ANSI/API-12J using CFD and finding that perforated plates and inlet diverters increased the efficiency of separation. In addition, they found that the efficiency of separation was sensible to the location of the perforated plates and inlet diverters. This conclusion is also found in important numerical computation studies; interested readers refer to [3]-[5].

The previous references [2]-[5] focused their CFD studies on a set of design conditions. Unfortunately, most of these devices work under transient conditions in large periods of the time and also off-design conditions were not considered. While CFD is becoming widely used for this analysis, it is also known that design must also consider off-design conditions. The focus of this study is three-fold. Firstly, we use CFD to validate the ANSI/API-12J 2008 edition for vertical-oil-gas separators. Secondly, we compare the recommended practices presented by [6]-[9] with the widely used international Standard ANSI/API-12J. Finally, we use multi-objective optimization and proxy models to find the best combination of design parameters that maximize the overall performance of the model. The Buckingham Pi theorem 
[10] is used to define the key design parameters based on operational considerations such as densities, mass flows rates and liquid droplet size. Design of experiment and proxy modeling techniques used were Latin hypercube and Kriging, respectively.

\section{VERTICAL OIL-GAS SEPARATOR DESIGN}

Vertical separators have four main sections, refer to Fig. 1. The primary section, located at the entrance of the separator, is used to separate the largest amount of liquid present in the flow. Normally, the inlet has a diverter plate that takes advantage of the difference in the momentum separating the heavier fluid. The secondary section, called gravity settling section, takes advantage of the density difference between the phases. The lighter particles suspended in gas stream settle out of the stream of gas if the velocity of the gas is sufficiently slow. The third section consists of specialized devices that allow the coalescence of the droplets, also known as mist extractors.

In this study, the size of the separator was determined for the oil-gas mixture properties shown in Table 1. Since the International Standard ANSI/API-12J does not provide a strict rule to define the length, this study used the general recommendation suggested by the ANSI/API-12J, and the length was fixed at 120 inches.

Table 2 shows the criteria used in this study to define the diameter of the separator. As it is shown in Table 2, the references used in this study define the terminal velocity based on the density difference between the gas and liquid.

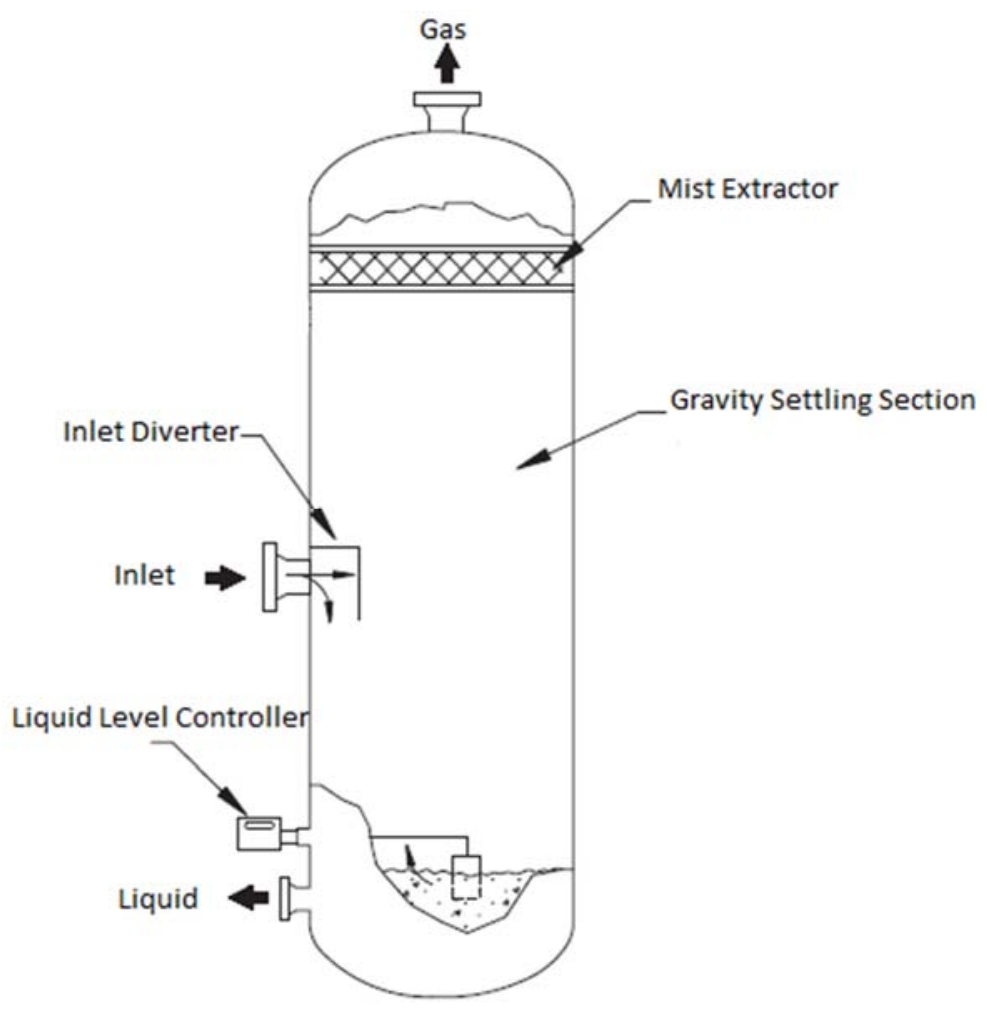

Figure 1: Schematic view of a typical vertical separator [6]. 
Table 1: Fluids properties.

\begin{tabular}{|l|l|}
\hline Gas flow rate & $25 \mathrm{MMSCFD}$ \\
\hline Liquid flow rate & $3000 \mathrm{BPD}$ \\
\hline Operating pressure & $800 \mathrm{psig}$ \\
\hline Surface tension & $25.050 \mathrm{dyn} / \mathrm{cm}$ \\
\hline Gas density (process condition) & $3.40 \mathrm{lb} / \mathrm{ft}^{3}$ \\
\hline Liquid density & $51.5 \mathrm{lb} / \mathrm{ft}^{3}$ \\
\hline Gas viscosity & $0.018 \mathrm{cp}$ \\
\hline Liquid viscosity & $2.2 \mathrm{cp}$ \\
\hline K factor (apply only to ANSI/API-12J) & 0.3 \\
\hline Operating temperature & $80^{\circ} \mathrm{F}$ \\
\hline Residence time & $1 \mathrm{~min}$. \\
\hline Gas compressibility factor & 0.820 \\
\hline Droplet size & $100 \mathrm{microns}$ \\
\hline
\end{tabular}

Table 2: Design criteria based on the different authors.

\begin{tabular}{|c|c|c|}
\hline Author & Terminal velocity & (eqn) \\
\hline ANSI/API-12J & $\left|\overline{\mathrm{v}}_{\mathrm{t}}\right|=\mathrm{K} \sqrt{\frac{d_{l}-d_{g}}{d_{g}}}$ & (1) \\
\hline Stewart and Arnold [6] & $\left|\bar{v}_{t}\right|=0.0119\left[\left(\frac{d_{l}-d_{g}}{d_{g}}\right) \frac{D_{p}}{C_{d}}\right]^{1 / 2}$ & (2) \\
\hline Campbell et al. [7] & $\left|\bar{v}_{t}\right|=\left[\frac{4 g D_{p}^{N+1}\left(d_{l}-d_{g}\right)}{3 A \mu^{N} d_{l}^{(1-N)}}\right]^{\left(\frac{1}{2-N}\right)}$ & (3) \\
\hline GPSA [8] & $\left|\bar{v}_{t}\right|=\sqrt{\frac{4 g D_{p}\left(d_{l}-d_{g}\right)}{3 d_{g} C^{\prime}}}$ & (4) \\
\hline Manning and Thompson [9] & $\begin{array}{l}\left|\overline{v_{t}}\right| \\
=\left(\sqrt{\left(4 D_{p} g / 3 C_{d}\right)}\right)\left(\sqrt{\left(d_{l}-d_{g}\right) / d}\right.\end{array}$ & (5) \\
\hline
\end{tabular}

Where $\overline{v_{\mathrm{t}}}$ represents the terminal velocity, $d_{l}$ is the density of the liquid and $d_{g}$ is the density of the gas. The diameter of the droplet is $D_{p}, C_{d}$ represents the drag coefficient, $C^{\prime}$ is the corrected drag coefficient, $g$ is gravity acceleration, $\mu$ represents the viscosity of the liquid, the coefficients $\mathrm{A}$ and $\mathrm{N}$ depend on the flow regime and the drag coefficient. It is important to mention that the references cited in this study concluded that these approaches are based on empirical correlations that do not include important effects such as vortices and temporal fluctuations and most of them do not consider turbulent regimes. As a result, uncertainties are present in such designs and reflected on different empirical factors such as 
"K" factor implemented in the ANSI/API-12J. In addition, they suggest to not replace the good judgment of the designer and complement the designs with experimental data.

Table 3 shows the diameter predicted by the references and equations listed in Table 2, considering the properties and conditions presented in Table 1.

Although the properties of the fluids are the same and the terminal velocity considers the difference of the density between the fluids, it is seen that these references yielded to different diameters. Nevertheless, the International Standard ANSI/API-12J suggests this methodology for vertical separator with mist extractors, which is not considered in this numerical study. These results demonstrate the importance of using an independent technique to validate these approaches and CFD is used in this study to evaluate each technique.

\section{COMPUTATIONAL FLUID DYNAMICS}

There are two main approaches to model multiphase fluid flows, the Eulerian and Lagrangian models. The Eulerian model resolves each phase directly and the interaction between the phases is considered through source terms in the general equations. In contrast, the Lagrangian model exclusively resolves the continuous phase, whereas the dispersed phase is calculated through coupling models. Both models resolve the continuous phase using the Eulerian model.

Typically, the dispersed phase is carried by the continuous phase without affecting the hydrodynamic from the continuous phase. Under this assumption, the continuous phase is resolved using a Eulerian frame of reference, based on the conservation equations from fluid dynamics, whereas the dispersed phase is solved under a Lagrangian frame of reference, using Newton's equations for each particle. Thus, the effects of the dispersed phase on the continuous phase is negligible. Consequently, this formulation is valid when the size of particles and the volumetric fraction of the dispersed phase are small [11]. This approach is also known as Euler-Lagrangian and it is valid when the volume fraction of the dispersed phase is lower than $10 \%$ [12]. Regardless of the type of model used to represent the dispersed phase, both phases interact by means of interfacial forces between their boundaries such as drag forces, buoyancy forces, virtual mass and turbulent dispersion.

Eqns (6) and (7) describe dynamics of incompressible flows in Cartesian coordinates and they represent conservation of mass and momentum, respectively. Heat transfer and radiation are neglected in this study, and thus, conservation of energy is not c.

$$
\begin{gathered}
\frac{\partial u_{i}}{\partial x_{i}}=0 \\
\frac{\partial u_{i}}{\partial t}+u_{j} \frac{\partial u_{i}}{\partial x_{j}}=-\frac{1}{\rho} \frac{\partial p}{\partial x_{i}}+\nu \nabla^{2} u_{i}+F_{i},
\end{gathered}
$$

Table 3: Predicted diameters.

\begin{tabular}{|l|c|c|}
\hline Author & Diameter (inches) $\left(\mathrm{D}_{\mathrm{v}}\right)$ & Hight (inches) $(\mathrm{L})$ \\
\hline ANSI/API-12J & 30 & 120 \\
\hline Stewart and Arnold [6] & 40 & 120 \\
\hline Campbell et al. [7] & 44 & 120 \\
\hline GPSA [8] & 44 & 120 \\
\hline Manning and Thompson [9] & 36 & 120 \\
\hline
\end{tabular}


where $p$ represents the static pressure, $\rho$ is the density of the continuous phase and $v$ is the kinematic viscosity of the continuous fluid. In addition, $x_{i}$ is the spatial direction, $u_{i}$ is the continuous phase velocity in the " $i$ " direction and $F_{i}$ describes momentum sources due to external body forces and any other momentum source.

The dispersed phase is solved by tracking many parcels throughout the domain. Eqn (8) is used to calculate the droplet velocity at each solved position

$$
m_{p} \frac{d u_{p}}{d t}=F_{\text {all }}
$$

Here $m_{p}$ is the mass of the droplet, $u_{p}$ the velocity of the particle and $F_{\text {all }}$ represents the sum of all forces acting on a particle. This study considers drag forces between phases, buoyancy forces due to gravity and turbulent dispersion forces. The latter is necessary because it generates additional dispersion of the phase from the high volumetric fraction region to the low one, due to turbulent fluctuations, additional information about the turbulence dispersion force can be found in [13]. Turbulence was resolved using the Reynolds Stress Model (RSM) because of the strong swirling and the anisotropic character of this type of flow configurations.

\subsection{Numerical study}

Each design is validated using CFD where the governing equations are discretized and solved along the boundary conditions. The computational mesh is developed following the good practices, where the quality was kept bellow 0.35 and the maximum allowed skewness was 0.8 . Firstly, inflation layers were placed near the wall and tetrahedral elements outside the wall region. Although it is not shown in this study, a consistent grid sensitivity analysis was performed and the results shown here represent the grid independent solution under the conditions specified in this study.

The numerical models implemented in this study are shown in Table 4. In this developmental stage, a symmetric boundary condition was imposed on the symmetry plane to save computational time, however, this condition is not strictly enforced under this configuration. The bottom outlet (liquid outlet) static pressure was defined at $0.37 \mathrm{psi}$ corresponding to the hydrostatic pressure at that location, whereas the static pressure at the top outlet (gas outlet) was fixed at 0 psi. No-slip is imposed on the walls, inlet, and outlet pipes. The restitution coefficients for the liquid phase were defined as 0 and 1 , perpendicular and parallel to the wall, respectively. The inlet boundary conditions were defined as follows: gas mass flow rate was $7.75 \mathrm{lb} / \mathrm{s}$ and $5.02 \mathrm{lb} / \mathrm{s}$ for the liquid phase. Normal velocity was defined only for the liquid phase at $0.56 \mathrm{ft} / \mathrm{s}$ and 15,000 particles were injected at the inlet with a random pattern.

Table 4: Numerical models.

\begin{tabular}{|l|l|}
\hline Reference pressure & $800 \mathrm{psi}$ \\
\hline Type of study & Steady state \\
\hline Fluids & Two fluids, gas and liquid \\
\hline Drag model & Ishii Zuber \\
\hline Coupling model & Full coupling \\
\hline Collision of particles & Sommerfield model (coefficients $=0$ ) \\
\hline
\end{tabular}


Figs 2 and 3 show the streamline colored by the velocity magnitude and averaged velocity on the upper head of the separator, respectively. Fig. 3 represents the averaged velocity for the ANSI/API-12J standard. For all cases, the averaged predicted velocity was smaller than the allowable velocity predicted by eqns (1)-(5) and the ANSI/API-12J yielded the largest averaged velocity.

The performance of these devices is evaluated using two variables, namely, the efficiency of separation and the dimensionless pressure drop, the latter known as the Euler number. These expressions are shown in eqns (9)-(10), respectively

$$
\begin{gathered}
\eta=\left(\frac{\dot{m}_{\text {lin }}-\dot{m}_{\text {lout }}}{\dot{m}_{\text {lin }}}\right) x 100 \\
E u=\frac{\Delta P}{d_{g} V_{\text {in }}^{2}},
\end{gathered}
$$

where $\dot{m}_{l}$ represents the mass flow rate of liquid and $\mathrm{V}_{\text {in }}$ is the inlet velocity of the continuous phase. Figs 4 and 5 depict the efficiency of separation and the Euler number for the different standards, respectively. Fig. 4 demonstrates that the efficiency of separation for all standards is greater than $99 \%$. However, the ANSI/API-12J delivered the lowest efficiency, which translates to annual loss of 992,077.67 lbs of liquid heavy hydrocarbon. To reduce the amount of liquid carried over by the continuous phase mist extractors are required. However, these devices impose extra pressure drop and this scenario is not considered in this study. Similarly, Fig. 5 shows that the ANSI/API provides one of the largest dimensionless pressure drop. It is important to mention that these findings agree with the predicted averaged velocity; where the largest efficiency is found for cases where the predicted velocity was the smallest. These preliminary results demonstrate that the International Standard ANSI/API-12J provides the less efficient design, whereas other techniques seem to be more efficient under the same boundary conditions and fluid properties. Nevertheless, those references are not considered International Standards and they suggest to consider the ANSI/API-12J as a design reference. In addition, these results are exclusively for one specific case and no conclusion can be drawn for different conditions, for example, different density ratios, droplet sizes and ratios between mass flow rates between continuous and dispersed phases. The following sections address this issue.

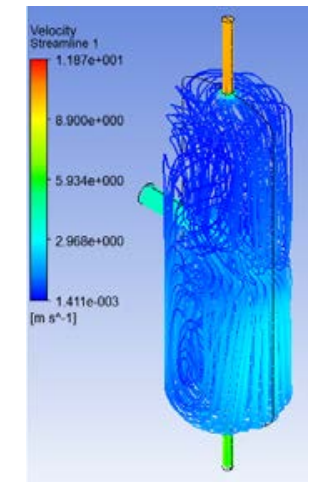

Figure 2: Streamlines.

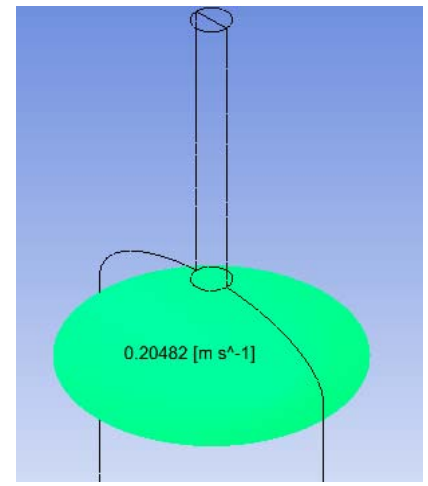

Figure 3: Averaged velocity. 


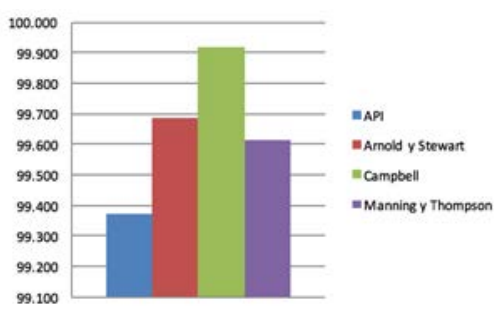

Figure 4: The efficiency of separation.

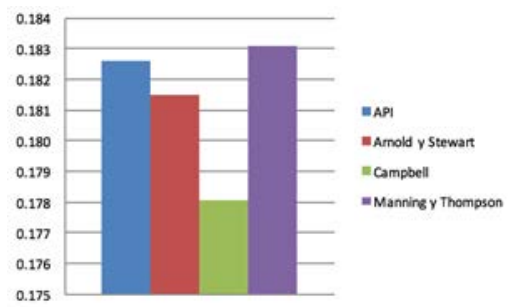

Figure 5: Dimensionless pressure drop.

\section{PROXY MODELLING AND OPTIMIZATION}

\subsection{Design parameters and performance measures}

In this preliminary stage, we introduce dimensionless parameters to characterize the phenomenon under consideration. This research assumes two independent variables: efficiency of separation and pressure drop. Thus, we can assume this problem can be modelled as:

$$
(\eta, \Delta P)=f\left(d_{g}, d_{l}, \dot{m}_{l}, \dot{m}_{g}, D_{p}, D_{v}\right) .
$$

The previous equation can be re-written in terms of dimensionless parameters obtained using the Buckingham Pi theorem [10] as:

$$
(\eta, E u)=f\left(\pi_{1}, \pi_{2}, \pi_{3}\right),
$$

where each dimensionless parameter is:

$$
\begin{gathered}
\pi_{1}=\frac{\dot{m}_{l}}{\dot{m}_{g}}, \\
\pi_{2}=\frac{d_{l}}{d_{g}}, \\
\pi_{3}=\frac{D_{p}}{D_{v}} .
\end{gathered}
$$

\subsection{Design of experiment}

To define the value of the variables $\pi_{i}$ Latin hypercube sampling is used and its solution is normalized based on the upper and lower bound specified for each parameter. These boundaries respond to operational and designs constraints. The lower bound for $\pi_{1}, \pi_{2}$ and $\pi_{3}$ were $0,11.76$ and 0 respectively. Whereas the upper bound was specified as $1.29,16.58$ and 0.000393 for $\pi_{1}, \pi_{2}$ and $\pi_{3}$, respectively. In this developmental stage, 24 samples were used although more samples are required [14], [15]. 
Each of the 24 samples is simulated and a value for each of the performance measures $(\eta, E u)$ was obtained. In other words, $\pi_{1}, \pi_{2}$ and $\pi_{3}$ are the inputs for the CFD case, whereas the efficiency of separation and Euler number are the outputs.

\subsection{Proxy modelling}

The proxy modelling is built from the solution of the 24 samples using Kriging because of its good properties with nonlinear problems and its capabilities of filtering noise from the input variables [14]. The objective of this section is to find the best combination of $\pi_{1}, \pi_{2}$ and $\pi_{3}$ that deliver the highest efficiency of separation while providing the lowest pressure drop. To this end, two surrogate models were obtained, one for the efficiency of separation and the other for pressure drop.

An important parameter to define the quality of the surrogate model is the coefficient of determination $r^{2}$. If the coefficient is equal to 1 , the surrogate model reproduces the test data perfectly, whereas values close to zero indicate there is no correlation between the surrogate model and the test data. In this case, the coefficient of determination was 0.60 for the efficiency of separation with a RMS of 1.067 and 0.80 for the Euler number; the latter with a RMS of 0.0029 . These coefficients are notably low, confirming the conclusion from [15] that suggested 20 samples per variable to obtain correlations close to 0.90 .

Figs 6 and 7 depict the response surface for the efficiency of separation and dimensionless pressure drop, respectively. This graphical presentation is recommended since $\pi_{3}$ represents the ratio between the liquid drop diameter and the separator diameter. In this case, the diameter of the separator was constant for all cases, whereas the diameter of the drop was changed. This condition is very common because of the transient characteristic of these types of flow configuration.

Fig. 6 shows the efficiency of separation remains almost constant regardless of the value of densities and mass flows, for large drop sizes. In contrast, the Euler number shows a similar trend for the different values of $\pi_{3}$. In other words, the diameter of the particle does not affect Euler number drastically, whereas the mass flow rate of the dispersed phase affects the pressure drop considerably. In conclusion, the Euler number is not an appropriate variable to assess the performance of these devices since it remains almost constant $(0.165-0.192)$, regardless of the mass flow rates and densities. Therefore, it is important to explore the effect of the $\pi_{1}, \pi_{2}$ and $\pi_{3}$ on the efficiency of separation.

Figs 8 and 9 depict the efficiency of separation for drop sizes between 100 and 300 microns $\left(9.5 \times 10^{-5}<\pi_{3}<1.6 \times 10^{-4}\right)$. As the diameter of the drop decreases, the efficiency of separation declines. In addition, Fig. 9 shows the effect of the mass flow ratios on the efficiency of separation, i.e. for $\pi_{3}=9.5 \times 10^{-5}$ the maximum efficiency is achieved when
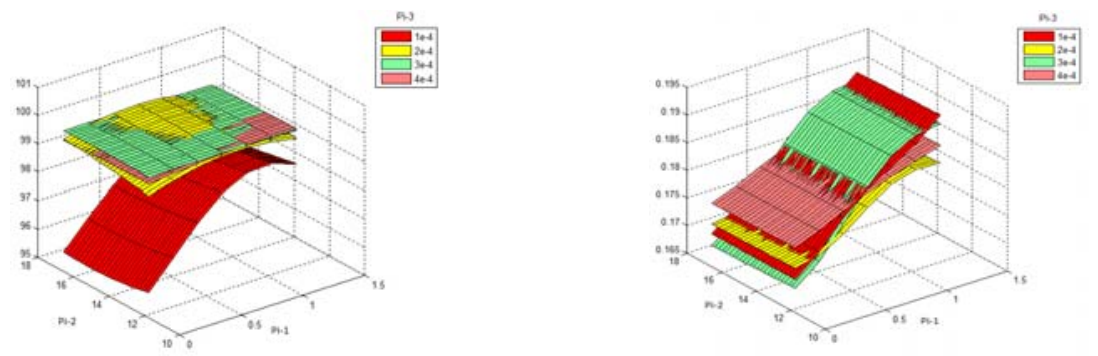

Figure 6: Response surface for the Figure 7: Response surface for the efficiency of separation. pressure drop. 


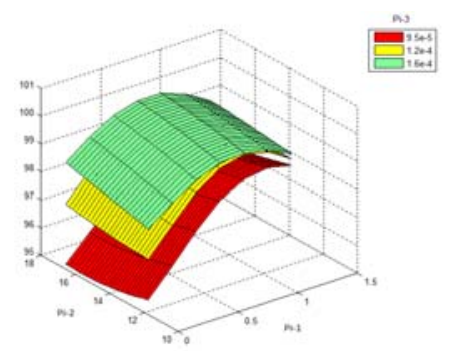

Figure 8: Response surface for the efficiency of separation for intermediate drop size.

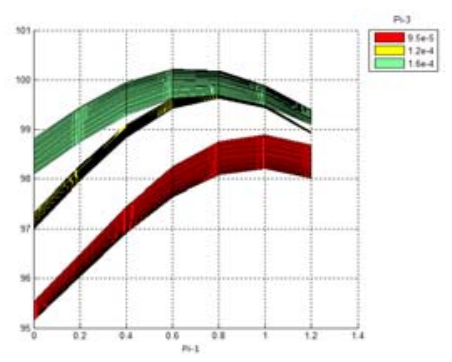

Figure 9: Response surface for the efficiency of separation for intermediate drop size (front view).

the mass flow rate of liquid and gas are the same. However, it is also demonstrated that the efficiency of separation reduces as the mass flow rate from the dispersed phase decreases. This solution contradicts the physical expectation since it is expected to obtain larger efficiencies as the mass flow rate of the dispersed phase decreases. However, full coupling was always used even for low values of $\pi_{1}$ and the number of particles used in this study is considerably small $(15,000)$. Furthermore, Figs 8 and 9 demonstrate that the efficiency of separation increases as the density of the dispersed phase increases agreeing with the physical expectations. From these two graphs, we can conclude that $\pi_{3}$ (drop size) plays the most important role, which confirms the need of using mist extractors for drop size smaller than 100 microns. Finally, Fig. 9 shows that the efficiency of separation reaches values above $100 \%$ which is technically impossible. This technical discrepancy comes as a result of the low determination coefficient mentioned before.

\subsection{Optimization}

Each of these proxy models are optimized using a stochastic global optimization method [16] Therefore, values of $\pi_{1}, \pi_{2}$ and $\pi_{3}$ that maximize the efficiency of separation and pressure drop are obtained, refer to Table 5 .

Table 5 shows two important features. Firstly, the optimized efficiency of separation is greater than 100 and this technical issue was addressed previously. Secondly, the value $\pi_{2}$ is the same for both models. This shows that $\pi_{2}$ does not play a dominant role compared to $\pi_{1}$ and $\pi_{3}$. However, this conclusion is only valid based on the parameters used in this study, and as it was suggested before, it is required to use more samples to obtain a higher coefficient of determination.

Table 5: Optimized values.

\begin{tabular}{|c|c|c|}
\hline & $\begin{array}{c}\text { Surrogate model for the } \\
\text { efficiency of separation }\end{array}$ & $\begin{array}{c}\text { Surrogate model for } \\
\text { Euler Number }\end{array}$ \\
\hline Function value & $\mathbf{1 0 0 . 2 6}$ & $\mathbf{0 . 1 6 8}$ \\
\hline$\pi_{1}$ & 0.6988 & 0.03392 \\
\hline$\pi_{2}$ & 16.439 & 16.439 \\
\hline$\pi_{3}$ & 0.000148 & 0.000318 \\
\hline
\end{tabular}




\subsection{Multi-objective optimization}

To maximize the efficiency of separation and the dimensionless pressure (Euler number) simultaneously, a multi-objective function was created, refer to eqn (14).

$$
\text { Objfun }=W_{1} \frac{\text { Efficiency }}{\text { Optimized Efficiency }}+W_{2} \frac{\text { Optimized Euler }}{\text { Euler }} \text {, }
$$

where $\mathrm{W}_{1}$ and $\mathrm{W}_{2}$ are weighting factors. A new surrogate model is built where output is the objective function shown in eqn (14), and whose inputs are the same $\pi_{i}$. The new surrogate model is optimized using the same stochastic global optimization method [16] and the solution is plotted in a Pareto chart, which is constructed changing the weighting factors, refer to Fig. 10.

Fig. 10 shows two fundamentals aspects of this phenomenon. Firstly, both parameters tend to the same direction and they do not compete. Thus, high efficiency of separation is found in regions where the Euler number is small. This finding agrees with previous comments where we stated that pressure drop does not play an important role in this phenomenon, and thus other parameters should be used to assess the performance of such devices. Fig. 10 shows that the variation in pressure drop is between 0.175 and 0.168 ; whereas the efficiency of separation varies between roughly between 91 and 100\%. Again, more sampling is required. It is worth mentioning that the points shown in Fig. 10 represent optimized points of operation for different weighting factors. Therefore, the goal is to determine the best combination that yields to the most efficient condition based on both outcomes (pressure drop and efficiency of separation). Under the conditions described in this study, the most efficient combination is represented by the gray zone in Fig. 10.

However, our analysis was focused on the dimensionless parameters $\pi_{i}$ and as a result, it is required to extract the best combination of $\pi_{1}, \pi_{2}$ and $\pi_{3}$ that yield to the best scenario. Table 6 shows the combination of $\pi_{1}, \pi_{2}$ and $\pi_{3}$ that represent the gray zone depicted in Fig. 10.

Table 6 demonstrates that the objective function (eqn (14)) is very sensitive to the ratio of the drop diameter to the separator diameter, in contrast to the ratio of mass flow rates and densities. It is also demonstrated and corroborated that the efficiency of separation increases as the drop size increases $\pi_{3}$.

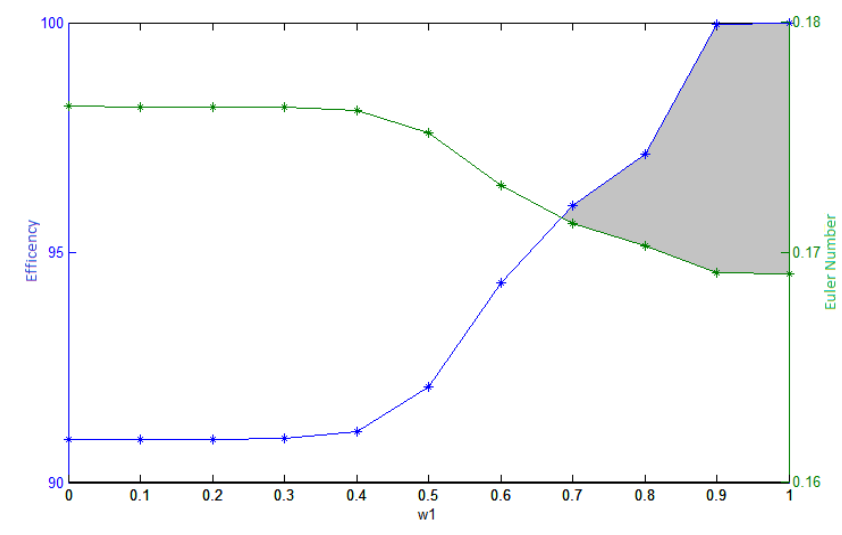

Figure 10: Multi-objective optimal values of efficiency of separation and Euler number for each weight combination. 
Table 6: Optimized input variables.

\begin{tabular}{|c|c|c|}
\hline$\pi_{1}$ & $\pi_{2}$ & $\pi_{3}$ \\
\hline 0.03392 & 16.439 & 0.0000437 \\
\hline 0.03392 & 16.439 & 0.0000439 \\
\hline 0.03392 & 16.439 & 0.0000442 \\
\hline 0.03392 & 16.439 & 0.0000459 \\
\hline 0.03392 & 16.439 & 0.0000517 \\
\hline 0.03392 & 16.439 & 0.0000659 \\
\hline 0.03392 & 16.439 & 0.0000864 \\
\hline 0.03392 & 16.439 & 0.0001017 \\
\hline 0.03392 & 16.439 & 0.0001150 \\
\hline 0.03392 & 16.350 & 0.0003059 \\
\hline 0.03392 & 16.439 & 0.0003091 \\
\hline
\end{tabular}

\section{CONCLUSIONS AND FUTURE WORK}

A detailed investigation of a typical oil-gas separator designed was performed using CFD. The CFD results shown in this study represent the grid independent solution and under the conditions presented here the international standard ANSI/API-12J delivered the lowest efficiency of separation. This validation demonstrates the pressure drop does not play an important role in assessing the performance of such devices. It was also demonstrated that the amount of liquid carried over by the continuous phase can be considerably high, leading to operation issues with the downstream equipment or loss of heavy hydrocarbons. Proxy Modeling was implemented and it allowed to understand the interaction between the different dimensionless parameters derived for this study. It was demonstrated that the efficiency of separation was sensible to the ratio between the diameter of the droplet and the diameter of the separator. A consistent procedure was implemented to build the proxy modeling where Latin hypercube was used and a stochastics method was implemented to optimize the parameters. The optimization processes reinforced the initial CFD conclusions about the pressure drop. We demonstrated that pressure drop and efficiency of separation tends toward the same direction. This phenomenon is evident since larger diameters yield to lower pressure drop and higher efficiency of separation. As a result, more samples are needed to obtain a larger coefficient of determination.

\section{ACKNOWLEDGEMENTS}

The authors thank Maida Bermudez Bosch MA, CCC-SLP for her help on editing and proofreading the written article, British Institution of Mechanical Engineers (IMechE) for their financial support. Also, the authors thank the important feedback and suggestions provided by the reviewer.

\section{REFERENCES}

[1] Laleh, A.P., Svrcek, W.Y. \& Monnery, W.D., Design and CFD studies of multiphase separators: A review. The Canadian Journal of Chemical Engineering, 90(6), pp. 1547-1561, 2012.

[2] Efendioglu, A., Mendez, J. \& Turkoglu, H., The numerical analysis of the flow and separation efficiency of a two-phase horizontal oil-gas separator with an inlet diverter and perforated plates. Advances in Fluid Mechanics, 10, p. 133, 2014. 
[3] Lu, Y., Lee, J.M., Phelps, D. \& Chase, R., eds, Effect of Internal Baffles on Volumetric Utilization of an FWKO - A CFD Evaluation. SPE Annual Technical Conference and Exhibition, Society of Petroleum Engineers, 2007.

[4] Lee, C.-M., van Dijk, E., Legg, M., Byeseda, J., eds, Field confirmation of CFD design for FPSO-mounted separator. Offshore Technology Conference, 2004.

[5] Hansen, E., Heitmann, H., Laska, B., Loes, M., eds, Numerical Simulation of Fluid Flow Behavior Inside, and Redesign of a Field Separator. Proceedings of the 6th International Conference on Multiphase Production, Cannes, France, pp. 117-129, 1993.

[6] Stewart, M. \& Arnold, K.. Gas-Liquid and Liquid-Liquid Separators, Gulf Professional Publishing, 2008.

[7] Campbell, J.M., Lilly, L. \& Maddox, R. eds, Gas Conditioning and Processing, vol. 3, PennWell Conferences and Exhibitions, Houston, TX, 1999.

[8] GPSA, Gas Processors and Suppliers Association Engineering Data Book, GPSA: Tulsa, OK, 2004.

[9] Manning, F.S. \& Thompson, R.E., Oilfield Processing of Petroleum: Crude Oil, Pennwell Books, 1991.

[10] White, F.M., Fluid Mechanics. McGraw-Hill Book Company: Boston, 2003.

[11] Prosperetti, A. \& Tryggvason, G., Computational Methods for Multiphase Flow, Cambridge University Press, 2009.

[12] Liu, H.-F., Xu, J.-Y., Wu, Y.-X., Zheng, Z.-C., Numerical study on oil and water twophase flow in a cylindrical cyclone. Journal of Hydrodynamics, Series B, 22(5), pp. 832-837, 2010.

[13] Dehbi, A., Turbulent particle dispersion in arbitrary wall-bounded geometries: A coupled CFD-Langevin-equation based approach. International Journal of Multiphase Flow, 34(9), pp. 819-828, 2008.

[14] Wang, G.G. \& Shan, S., Review of metamodeling techniques in support of engineering design optimization. Journal of Mechanical Design, 129(4), pp. 370-380, 2007.

[15] Forrester, A.I.J., Bressloff, N.W. \& Keane, A.J., eds, Optimization using surrogate models and partially converged computational fluid dynamics simulations. Proceedings of The Royal Society A, 462, pp. 2177-2204, 2006.

[16] Csendes, T., Pál, L., Sendin, J.O.H. \& Banga, J.R., The GLOBAL optimization method revisited. Optimization Letters, 2(4), pp. 445-454, 2008. 